\title{
FPGA implementation of color image encryption using a new chaotic map
}

Hamsa A. Abdullah, Hikmat N. Abdullah

College of Information Engineering, Al-Nahrain University, Baghdad, Iraq

\begin{tabular}{l}
\hline \hline Article Info \\
\hline Article history: \\
Received Jul 13, 2018 \\
Revised Oct 2, 2018 \\
Accepted Oct 19, 2018 \\
\hline
\end{tabular}

\section{Keywords:}

FPGA

Image encryption

Nahrain chaotic map

Scrambling

Security analysis

\begin{abstract}
In this paper, an FPGA implementation of efficient image encryption algorithm using a chaotic map has been proposed. The proposed system consists of two phases image encryption technique. First phase consists of scrambling of pixel position and second phase consist of diffusion of bit value. In the first phase, original pixel values remain unchanged. In second phase, pixel values are modified. These modifications are done by using chaotic behavior of a recently developed chaotic map called Nahrain. A color image encryption using Nahrain chaotic map is simulated in software via Matlab, Altera Quartus Prime 17.0 Lite EditionI and ModelSim software tools then implemented in hardware via Cyclone V GX Starter Kit FPGA platform. The results show the feasibility and effectiveness of the cryptosystem. As a typical application, the image encryption/decryption is used to demonstrate and verify the operation of the cryptosystem hardware. Complete analysis on robustness of the method is investigated. Correlation, Encryption time, Decryption time and key sensitivity show that the proposed crypto processor offers high security and reliable encryption speed for realtime image encryption and transmission. To evaluate the performance, histogram, correlation, information entropy, number of pixel change rate (NPCR), and unified average changing intensity (UACI) measures are used for security analysis. The simulation results and security analysis have demonstrated that the proposed encryption system is robust and flexible. For example the amount of entropy obtained by the proposed algorithm is 7.9964, which is very close to its ideal amount: 8, and NPCR is $99.76 \%$, which is the excellent value to obtain. The hardware simulation results show that the number of pins that used of the proposed system reaches to $6 \%$ of total pins and Logic utilization (in ALMs) is $1 \%$.
\end{abstract}

Copyright $@ 2019$ Institute of Advanced Engineering and Science. All rights reserved.

\section{Corresponding Author:}

Hamsa A. Abdullah,

Department of System Engineering,

Al- Nahrain University, Baghdad, Iraq.

Email: hamsa.abdulkareem@coie-nahrain.edu.iq

\section{INTRODUCTION}

Chaotic system of simple structure can demonstrate complex dynamical properties in infinite mathematical world, such as sensitivity to initial conditions, topological transitivity and mixing, expansiveness, and decaying autocorrelation function. Chaotic system can be divided into two types of model: chaotic flow model and chaotic map model [1]. Such properties have subtle relation with some requirements of secure encryption system, especially sensitivity with respect to change of secret key [2-3]. So, designing chaos-based encryption schemes emerged as a new research direction to reinforce information security of data sent through the Internet.

Cryptography are realized in software to verify the effectiveness of algorithms other than in hardware for communication and storage applications. In fact, the main advantage of using of hardware for 
cryptosystem is that multiple parts of system can operate simultaneously, i.e. the generator of secret keys, substitution and permutation processes. That makes chaotic cryptosystems suitable for applications in high speed communications or massive storage [4]. In general, the encryption process conducted by using software that is programmed in computer. In practice, only a few applications requiring throughput while flexible solutions and low cost encryption / decryption is needed to protect the data that makes sense, especially for embedded hardware applications. Some small devices such as Field Programmable Gate Array (FPGA) have been potential to be applied to replace the computer as a medium for image encryption. The data encryption system will be optimized when implemented into the FPGA because it has advantages which include flexibility, development cost and costs low per-unit, high speed and has a good level of security [5].

In [4], an implementation of secure SPN chaos-based cryptosystem on FPGA is introduced. In this paper, the secure substitution permutation network (SPN) chaos-based cryptosystem is realized using software tools C/C++, Altera Quartus II and ModelSim and implemented in hardware using FPGA platform. In [5], the Sum of Product (SOP) Encryption using simple algorithm based on Boolean algebra is introduced. It is implemented using image encryption algorithm to produce a quick image encryption system. However it has drawback of having fixed encryption pattern. In [6], FPGA Implementation of Chaotic based AES Image Encryption Algorithm is presented. The algorithm is a combination of chaotic maps and AES. The proposed architecture is implemented using Verilog HDL and Xilinx ISE Design Suite 14.5. However it has drawback of having complex structures of image encryption.

In [7], Comparative analysis of color image encryption using 3D chaotic maps is presented. The color image encryption using different 3D chaotic maps, including 3D logistic map, Henon map, Baker map and cross chaos map which are the further extension of 2D chaotic maps. In [8], color image encryption using chaotic maps triangular scrambling, with DNA Sequences is introduced, The scheme takes a master key with a length of $320 \mathrm{bit}$, and produces a group of sub-keys with two length (32 and 128 bit) to encrypt the blocks of images, then a new triangular scrambling method is used to increase the security of the image. However it has drawback of having limited length of master key. In [9], Finite precision logistic map between computational efficiency and accuracy with encryption applications is introduced. Digital implementation of the generalized logistic map with signed parameter is considered. They present a fixed-point hardware realization of a pseudo-random number generator using the logistic map that experiences a trade-off between computational efficiency and accuracy. The trade-off factors include: the used precision, the order of execution of the operations, parameter, and initial point values affect the properties of the finite precision map. In [10], a color image encryption scheme based on arnold scrambling and quantum chaotic is introduced. In this paper, an algorithm for image encryption based on twodimensional Arnold transform with keys and quantum chaotic map is proposed.

In 2018, we have proposed a new dynamical system called Nahrain chaotic system [11]. We proved that it has excellent performance for multimedia encryption and secure transmission [12]. However, in [11] we have presented only a part standard randomness tests to prove the system randomness behavior. In this paper, we will introduce the hardware implementation of image encryption based on Nahrain chaotic map. The hardware implementation is based on a programmable hardware which allows the experimental characterization of the system dynamics with low cost, reconfigurable and rapid experimental setup. The paper is organized as follows. Section 1 presents presents the current stage of chaos based image encryption. Section 2 includes description of proposed encryption algorithm. Section 3 includes the simulation results and discussion of the proposed algorithm. Finally, section 4 gives the conclusions of the paper.

\section{IMAGE ENCRYPTION BASED ON CHAOTIC ALGORITHM}

The Block Diagram of the proposed image encryption algorithm based on Nahrain chaotic system is shown in Figure1. This encryption algorithm consists of two blocks: the first block is the Image Scrambling and second block is diffusion based on Nahrain Chaotic system. The nonlinear equations that describe the Nahrain chaotic system are given in (1):

$$
\begin{aligned}
& X_{n+1}=1-a X_{n} Y_{n}-X_{n}^{2}-Y_{n}^{2} \\
& Y_{n+1}=X_{n} \\
& Z_{n+1}=Y_{n}-b Z_{n}
\end{aligned}
$$

Where $\mathrm{a}$ and $\mathrm{b}$ are the bifurcation parameters of the system. Through a series of numerical modeling and simulation associated with MATLAB, The phase portraits of chaotic behavior have been acquired by using system parameter values: $a=1.52$ and $b=0.05$. The schematic block diagram of the proposed Nahrain chaotic generator is shown in Figure 1. Figure 2 shows the phase portraits of the proposed system when its initial 
conditions are: $\mathrm{X}(0)=0.3, \mathrm{Y}(0)=0.2$ and $\mathrm{Z}(0)=0.1$ [11]. The proposed scrambling method consist of two types of scrambling, the first method is Block Scrambling and the second method is negative diagonal scan. The main steps of encryption method are listed:

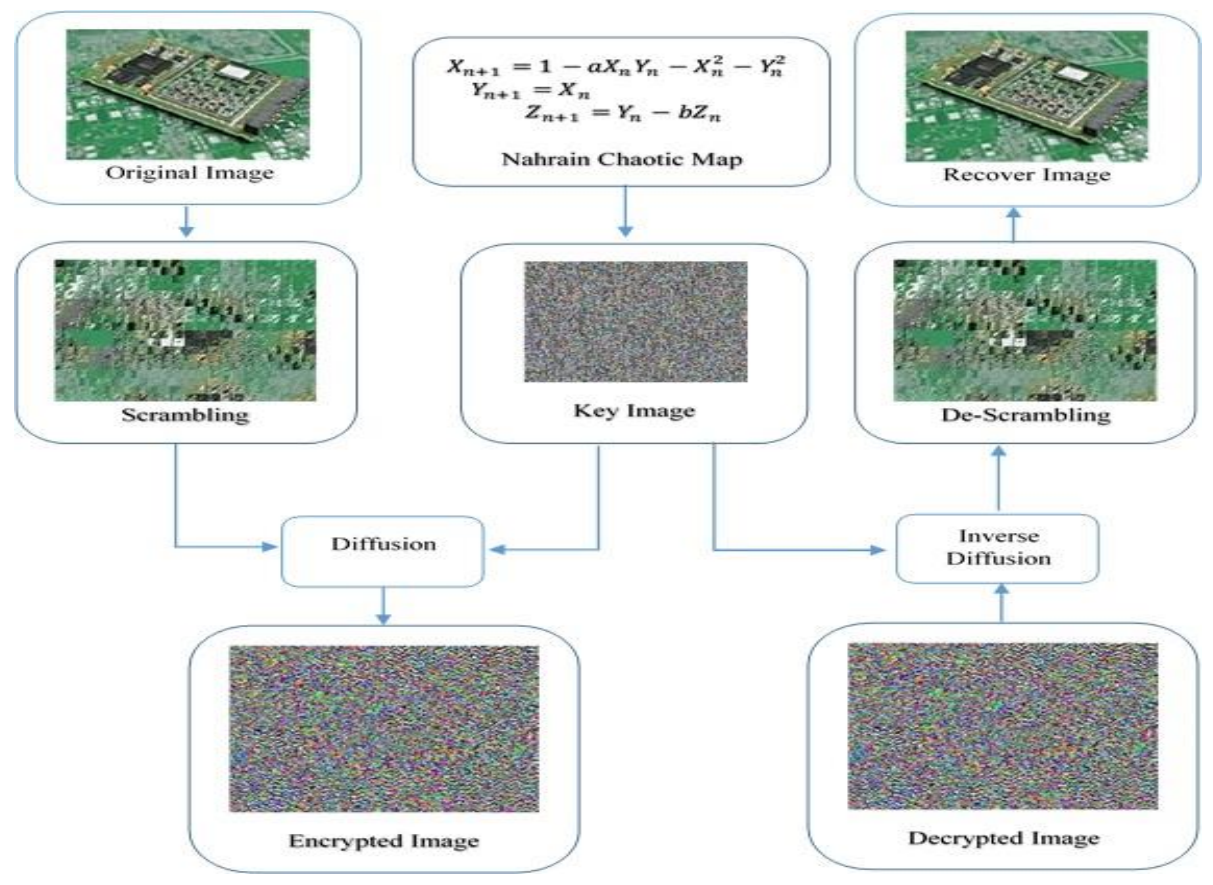

Figure 1. Proposed Algorithm

Step 1: Block Scrambling: the image is divided to four blocks [Block 1, Block2; Block3, Block 4] and then scrambling the blocks in the order [Block3, Block 4; Block1, Block2] as shown in Figure 2 (a).

Step 2: Each block is divided in to four sub block, and scrambled diagonally.

Sterp 3: Negative diagonal scan: the image is divided into blocks of size $8 * 8$ and then each block is read from right to left diagonally as shown in Figure 2 (b).

\begin{tabular}{|c|c|c|c|c|c|c|c|c|c|}
\hline \multirow[b]{2}{*}{$\begin{array}{c}\text { B1 } \\
\text { A }\end{array}$} & \multirow[b]{2}{*}{${ }^{B 2}$} & \multirow{3}{*}{$\Rightarrow$} & \multirow[b]{2}{*}{ B3 } & \multirow[b]{2}{*}{ B4 } & \multirow{2}{*}{ 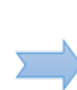 } & \begin{tabular}{|l}
$B 31$ \\
\end{tabular} & \begin{tabular}{|l|l} 
\\
\end{tabular} & B41 & \begin{tabular}{|l|l}
842 \\
\end{tabular} \\
\hline & & & & & & \begin{tabular}{|l}
$\mathrm{B} 33$ \\
\end{tabular} & 834 & \begin{tabular}{|l|l}
$B 43$ \\
\end{tabular} & 644 \\
\hline \multirow[t]{2}{*}{ B3 } & \multirow{2}{*}{$B 4$} & & \multirow{2}{*}{ B1 } & \multirow{2}{*}{ B2 } & & $B 21$ & B22 & B11 & $\beta 12$ \\
\hline & & & & & & $B 25$ & T:24 & B13 & $\Delta 14$ \\
\hline
\end{tabular}

\begin{tabular}{|l|l|l|l|}
\hline B34 & B33 & B44 & B43 \\
\hline B32 & B31 & B42 & B41 \\
\hline B24 & B23 & B14 & B13 \\
\hline B22 & B21 & B12 & B11 \\
\hline
\end{tabular}

(a). Block scrambling

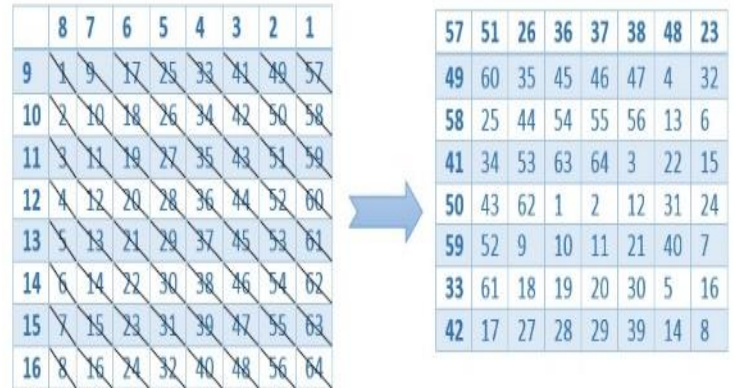

(b). Negative diagonal scrambling

Figure 2. Image scrambling

Step 4: Three dimensional chaotic map is generated by using (1).

Step 5: Chaotic (X1, Y1, and Z1) is converted to binary by using random bit generator method as shown [10]:

$$
B X(X 1, X 2)=\left\{\begin{array}{l}
1 \text { if } X 1>X 2 \\
0 \text { if } X 1 \leq X 2
\end{array}\right\} \text { where } X 1_{0} \neq X 2_{0}
$$




$$
\begin{aligned}
& B Y(Y 1, Y 2)=\left\{\begin{array}{l}
1 \text { if } Y 1>Y 2 \\
0 \text { if } Y 1 \leq Y 2
\end{array}\right\} \text { where } Y 1_{0} \neq Y 2_{0} \\
& B Z(Z 1, Z 2)=\left\{\begin{array}{l}
1 \text { if } Z 1>Z 2 \\
0 \text { if } Z 1 \leq Z 2
\end{array}\right\} \text { where } Z 1_{0} \neq Z 2_{0}
\end{aligned}
$$

The random bit generator method based on comparing the outputs of two Nahrain chaotic maps running side-by-side and starting from random independent initial conditions and same parameters value where $(\mathrm{X} 1=0.3, \mathrm{Y} 1=0.2, \mathrm{Z} 1=0.1, \mathrm{X} 2=0.2, \mathrm{Y} 2=0.1, \mathrm{Z} 2=0.2, \mathrm{a}=1.52$, and $\mathrm{b}=0.05)$.

Step 6: The output of row Scrambled image is XORed with the key image generated in steps (4 and 5) to get color level encrypted image.

The main steps of Decryption Method are listed below:

Step 1: Generate three dimensional chaotic map by using (1).

Step 2: Convert chaotic (X1, Y1, and Z1) to binary by using random bit generator method as shown in (2-4).

Step 3: The Encrypted image is XORed with key image generated in step 1 to get color level Scrambled image.

Step 4: Scrambled image is divided to block of size $8 * 8$ and then each block is read in negative diagonal scan as shown in Figure 2.b.

Step 5: The descrambled image is passed through Block Scrambling where image is divided into four blocks [Block 1, Block2; Block3, Block4] and then scramble the block in the order [Block3, Block4; Block1, Block2] as shown in Figure 2.a.

\section{RESULT AND DISCUSSION}

In this section, the simulation results of the security measurements for the proposed color image encryption using a new chaotic map is simulated in software via Matlab, Altera Quartus Prime 17.0 Lite Edition I and ModelSim software tools then implemented in hardware via Cyclone V GX Starter Kit FPGA platform. As a typical application, the image encryption/decryption is to demonstrate and verify the operation of the cryptosystem hardware. This project is implemented in two ways, namely.

a) By using MATLAB

b) By using FPGA

Here an VHDL block is used as an intermediate between the Matlab as shown in Figure 3, which means by the VHDL code we can't read the image directly so to overcome that one we use Matlab at the first, were an image is converted into text form and once more any random image is taken and converted into text form by Matlab, which acts as key image for the encryption this key should be same for encryption as well as decryption. Firstly the text file read by VHDL and then the two types of scrambling implemented on it. Finally an XOR operation is done between scrambled text matrix and key text matrix to get an encrypted result.

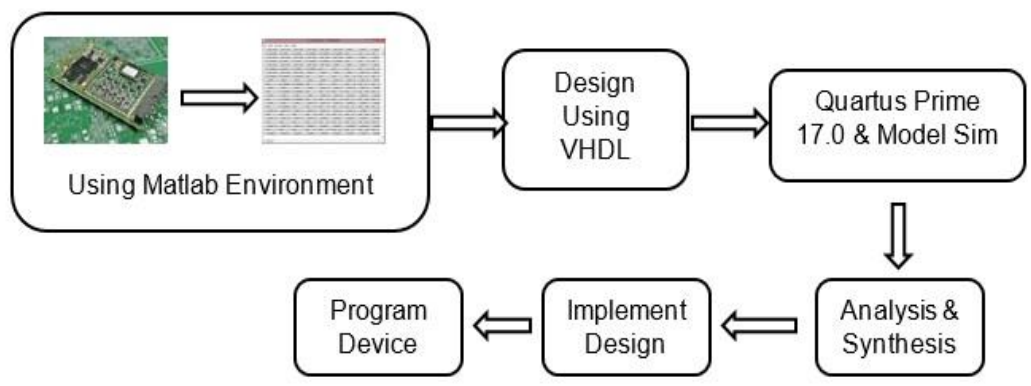

Figure 3. Hardware implementation steps

\subsection{MATLAB Implementation}

Color image of sizes $(64 \times 64,128 \times 128,176 \times 144$, and 256×256) are considered for experimental tests. The parameters of Nahrain map are taken to be $a=1.52, b=0.05$, and the initial conditions of state variables are $\mathrm{X} 0=0.3 ; \mathrm{Y} 0=0.2$; and $\mathrm{Z} 0=0.1$. Figure 4 shows the plain and encrypted images using Nahrain 
map. The security analysis of encrypted images is performed by using six statistical methods as: histogram analysis, PSNR, Information Entropy, Correlation, NPCR and UACI [12-14].
a. Original Image
b. Encrypted Image
$64 \times 64$

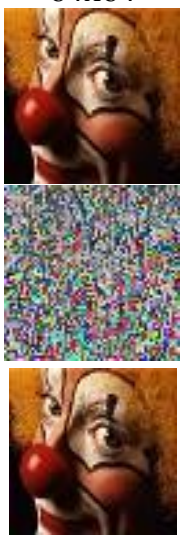
$128 \times 128$

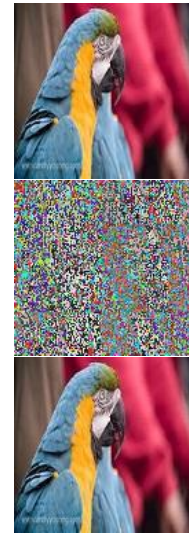
$176 \times 144$
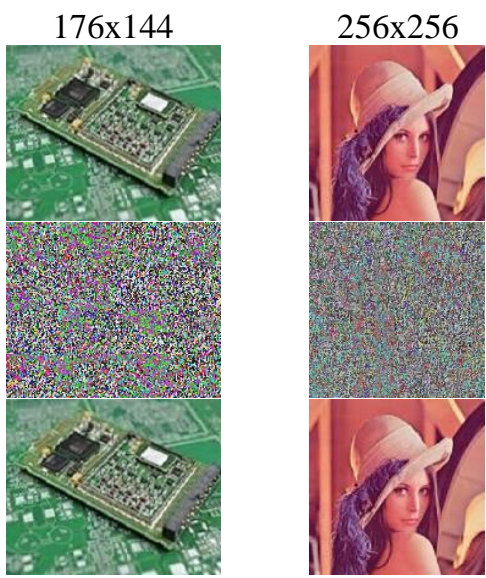

Figure 4. Plain and encrypted images

\subsubsection{Histogram Analysis}

Figure 5 shows the histogram analysis results. In Figure 5(a-e) are the histograms of color plainimage Red, Green, and Blue while (f-i) the histograms of encrypted-images Red, Green, and Blue using Nahrain chaotic map. As it is clearly seen, the histogram of proposed algorithm is fairly uniform and significantly different from that of the plain image and the encrypted image [4] of in Figure 5 (j). This reveals that the attacker cannot use any valid statistical data in the encrypted image to start any statistical attacks to the cryptosystem.

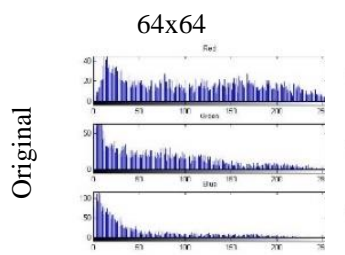

(a)

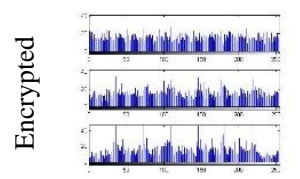

(f)

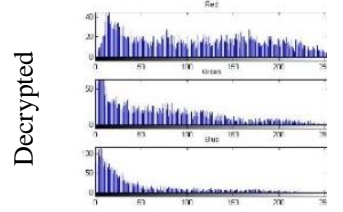

(k)

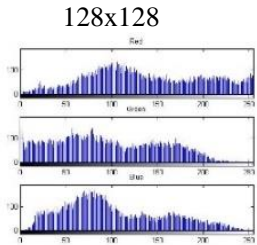

(b)

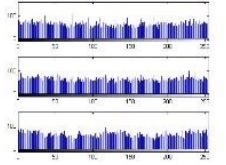

(g)

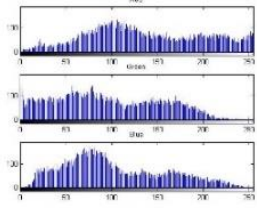

(1)

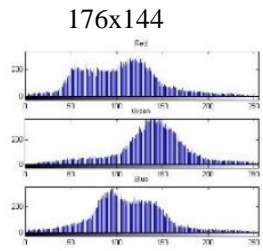

(c)

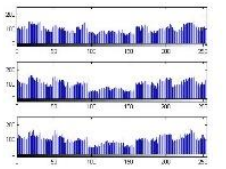

(h)

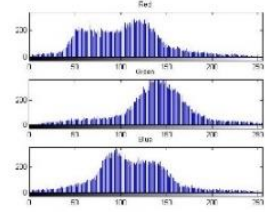

(m)

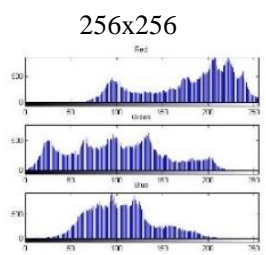

(d)

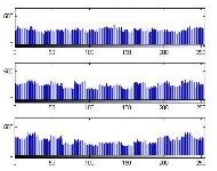

(i)

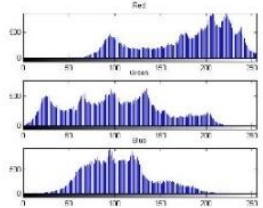

(n)

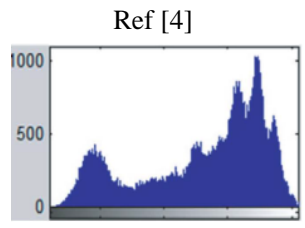

(e)

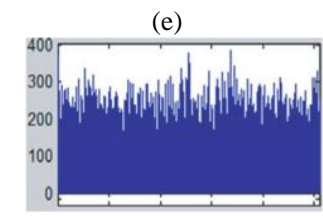

(j)

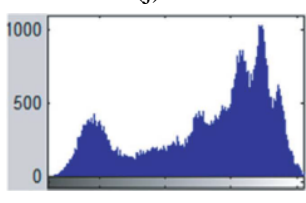

(o)

Figure 5. Histogram analysis

\subsubsection{PSNR}

Table 1 show the results of MSE and PSNR test between the original image, cipher image and reconstructed image. The comparison of PSNR values between the original image and the reconstructed 
image denoted that the two images are identical. Whereas the similarity PSNR values between the original image and cipher image are very low which means the encrypted image is very strong against the attacks.

\begin{tabular}{|c|c|c|c|c|c|}
\hline \multirow{2}{*}{ Image size } & \multirow{2}{*}{ Color layer } & \multicolumn{2}{|c|}{ Cipher Image } & \multicolumn{2}{|c|}{ Reconstructed Image } \\
\hline & & PSNR & MSE & PSNR & MSE \\
\hline \multirow{4}{*}{$64 \times 64$} & $\mathrm{R}$ & 12.2946 & $3.8337 \mathrm{e}+03$ & Inf & 0 \\
\hline & G & 12.0084 & $4.0949 \mathrm{e}+03$ & Inf & 0 \\
\hline & B & 11.3235 & $4.7943 e+03$ & Inf & 0 \\
\hline & $\mathrm{R}$ & 12.7008 & $3.4914 \mathrm{e}+03$ & Inf & 0 \\
\hline \multirow[t]{3}{*}{$128 \times 128$} & G & 12.6868 & $3.5027 \mathrm{e}+03$ & Inf & 0 \\
\hline & B & 12.9555 & $3.2926 \mathrm{e}+03$ & Inf & 0 \\
\hline & $\mathrm{R}$ & 13.1289 & $3.1637 \mathrm{e}+03$ & Inf & 0 \\
\hline \multirow[t]{3}{*}{$176 \times 144$} & G & 13.2544 & $3.0736 \mathrm{e}+03$ & Inf & 0 \\
\hline & B & 13.1379 & $3.1571 \mathrm{e}+03$ & Inf & 0 \\
\hline & $\mathrm{R}$ & 12.6553 & $3.5282 \mathrm{e}+03$ & Inf & 0 \\
\hline \multirow[t]{2}{*}{$256 \times 256$} & G & 12.9418 & $3.3029 \mathrm{e}+03$ & Inf & 0 \\
\hline & B & 13.2404 & $3.0835 \mathrm{e}+03$ & Inf & 0 \\
\hline
\end{tabular}

\subsubsection{Correlation Coefficients of Two Adjacent Pixels}

The correlation coefficients of plain and encrypted images are shown in Table 2. The correlation coefficients of encrypted images using the proposed algorithm are closed to zero, which means that the correlations of neighboring pixels in the plain image can be removed by using proposed algorithm, and can hold statistical attacks [15].

Table 2. Correlation Coefficients Results

\begin{tabular}{|c|c|c|c|c|c|}
\hline Algorithm & Image size & Color layer & Horizontal & Vertical & Diagonal \\
\hline \multirow{12}{*}{ Proposed Algorithm } & \multirow{3}{*}{$64 \times 64$} & $\mathrm{R}$ & 0.0121 & -0.0624 & 0.1083 \\
\hline & & G & -0.1360 & -0.1550 & 0.0919 \\
\hline & & B & -0.1171 & 0.0535 & 0.0319 \\
\hline & \multirow{3}{*}{$128 \times 128$} & $\mathrm{R}$ & -0.0214 & 0.0178 & 0.0586 \\
\hline & & G & 0.0779 & 0.0154 & -0.0032 \\
\hline & & B & 0.0016 & 0.0707 & -0.0373 \\
\hline & & $\mathrm{R}$ & -0.0525 & -0.0866 & -0.0757 \\
\hline & \multirow{3}{*}{$176 \times 144$} & G & 0.0239 & -0.1727 & 0.1079 \\
\hline & & B & -0.0553 & $7.1453 \mathrm{e}-04$ & -0.0448 \\
\hline & & $\mathrm{R}$ & 0.0079 & 0.0245 & 0.1259 \\
\hline & \multirow{2}{*}{$256 \times 256$} & G & -0.0921 & 0.0372 & 0.1013 \\
\hline & & B & -0.0410 & -0.0079 & 0.0316 \\
\hline Ref [7] & $256 \times 256$ & Average & 0.0124 & -0.00564 & 0.0276 \\
\hline \multirow[t]{2}{*}{$\operatorname{Ref}[8]$} & $256 \times 256$ & Average & 0.0007 & 0.0037 & -0.0051 \\
\hline & & $\mathrm{R}$ & -0.0071 & 0.0007 & 0.0014 \\
\hline \multirow[t]{2}{*}{$\operatorname{Ref}[9]$} & $256 \times 256$ & G & 0.0006 & 0.0006 & 0.0001 \\
\hline & & B & 0.0001 & 0.0045 & -0.0022 \\
\hline
\end{tabular}

\subsubsection{The Information Entropy}

The entropies of plain and encrypted images are shown in Table 3. The results in this table show that the entropies of the encrypted image generated by the proposed algorithm are closed to 8 , and that means the encrypted images are closed to a random.

\subsubsection{NPCR}

NPCR calculates the percentage of different pixel numbers between two Images. This analysis helps in determining the vulnerability of the system towards differential attacks. The analysis report for encrypted image is given in the Table 3. We note from this table that the proposed algorithm has the excellent performance where the value of NPCR is $99.74 \%$.

\subsubsection{UACI}

UACI calculate the average intensity of differences between two images. The analysis reported for encrypted image is given in the Table 3 . The value of UACI is over $33 \%$ which indicate that the rate of influence due to one pixel change is very large. 
Table 3. EC, NPCR and UACI Result

\begin{tabular}{|c|c|c|c|c|c|}
\hline Algorithm & Image size & Color layer & $\mathrm{EC}$ & NPCR & UACI \\
\hline \multirow{12}{*}{ Proposed Algorithm } & \multirow{3}{*}{$64 \times 64$} & $\mathrm{R}$ & 7.9503 & 99.66 & 34.81 \\
\hline & & G & 7.9342 & 99.49 & 34.59 \\
\hline & & B & 7.8427 & 99.73 & 40.24 \\
\hline & \multirow{3}{*}{$128 \times 128$} & $\mathrm{R}$ & 7.9692 & 99.54 & 32.33 \\
\hline & & G & 7.9774 & 99.62 & 33.46 \\
\hline & & B & 7.9666 & 99.64 & 31.94 \\
\hline & \multirow{3}{*}{$176 \times 144$} & $\mathrm{R}$ & 7.9064 & 99.66 & 32.31 \\
\hline & & G & 7.8169 & 99.76 & 32.91 \\
\hline & & B & 7.8271 & 99.74 & 33.14 \\
\hline & \multirow{3}{*}{$256 \times 256$} & $\mathrm{R}$ & 7.9964 & 99.58 & 32.60 \\
\hline & & G & 7.9628 & 99.65 & 32.19 \\
\hline & & B & 7.8294 & 99.74 & 32.70 \\
\hline $\operatorname{Ref}[7]$ & $256 \times 256$ & Average & 7.9437 & 98.92 & 33.50 \\
\hline $\operatorname{Ref}[10]$ & $256 \times 256$ & Average & - & 99.69 & 33.44 \\
\hline
\end{tabular}

\subsubsection{Key Space Analysis}

The size of the key space is the total number of different keys that can be applied in the encryption/ decryption process. The key space should be large enough to make brute-force attacks infeasible. From the cryptographic point of view, the size of the key space should not be smaller than 2100 to ensure a high level of security. The set of all initial numbers compose the key space. The key space of the image encryption scheme has five secret key values $\mathrm{x} 0, \mathrm{y} 0, \mathrm{z} 0$, a, and $\mathrm{b}$. As stated in IEEE floating-point standard, the computational precision of the 64-bit double-precision number is about 2-52 [16-18]. Therefore, the key space of the proposed algorithm is $(252) 5=2260$, which very much higher than the classical encryption schemes.

\subsection{FPGA Implementation}

The secure image encryption based on chaotic system has been implemented on the Altera FPGA Board, Cyclone V GX Starter Kit as shown in Figure 6 and 7. The color image with different sizes $(64 \times 64$, $128 \times 128,176 \times 144$, and $256 \times 256$ ) shown in Figure 4 are used to test the hardware implementation performance. The amount of time is required for encryption and decryption are shown in Table 4. The hardware resource required for the cryptosystem is considerably large due to simultaneous operation in key generator and encryption/decryption processes. The hardware resources that used shown in Table 5. The plain, Encrypted and Decrypted images obtained from the operation of cryptosystem on FPGA are shown in Figure 4(a-c). Decryption is exactly reverse to the encryption and final Encryption and Decryption simulation result as Figure 8.

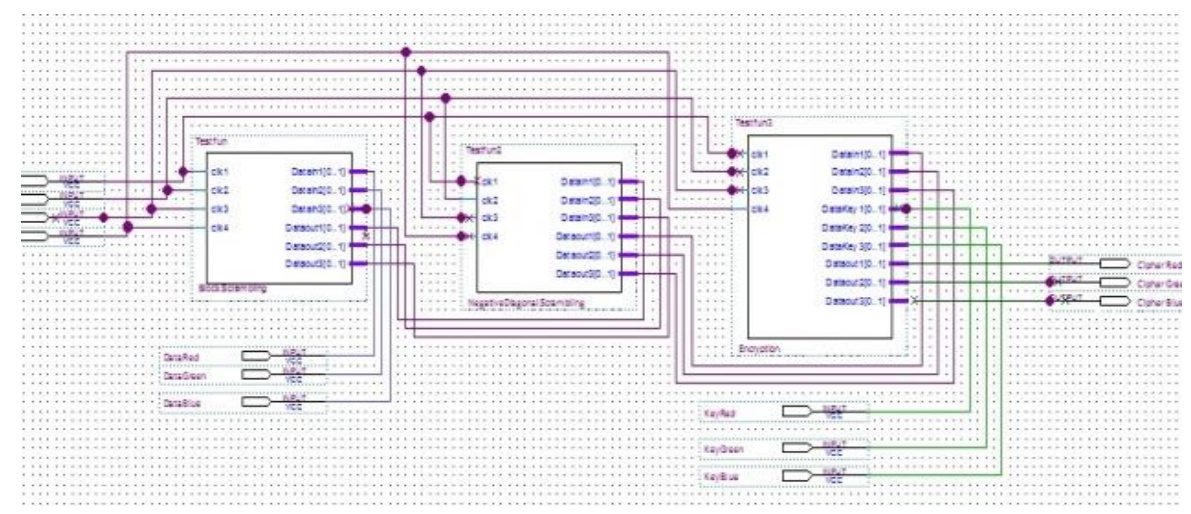

Figure 6. Block diagram of toe proposed system in FPGA

Table 4. Analysis \& Synthesis CPU Time

\begin{tabular}{ccc}
\hline Image size & Encryption time (in sec.) & Decryption time (in sec.) \\
\hline $64 \times 64$ & 0.44 & 1.16 \\
$128 \times 128$ & 1.20 & 1.24 \\
$176 \times 144$ & 1.55 & 2.04 \\
$256 \times 256$ & 4.14 & 4.35 \\
\hline
\end{tabular}




\begin{tabular}{cccc}
\multicolumn{4}{c}{ Table 5. Hardware Resources } \\
\hline & Total & Used & $\%$ \\
\hline Logic utilization (in ALMs) & 29,080 & 1 & $<1 \%$ \\
Pins & 364 & 22 & $6 \%$ \\
\hline
\end{tabular}

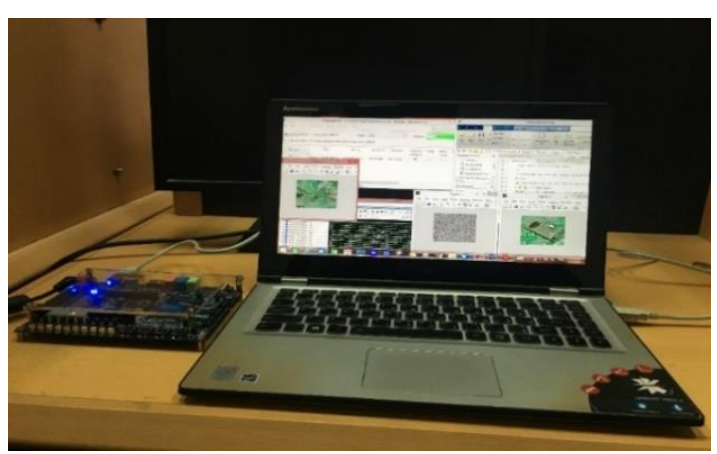

Figure 7. Altera FPGA board, cyclone V GX starter kit

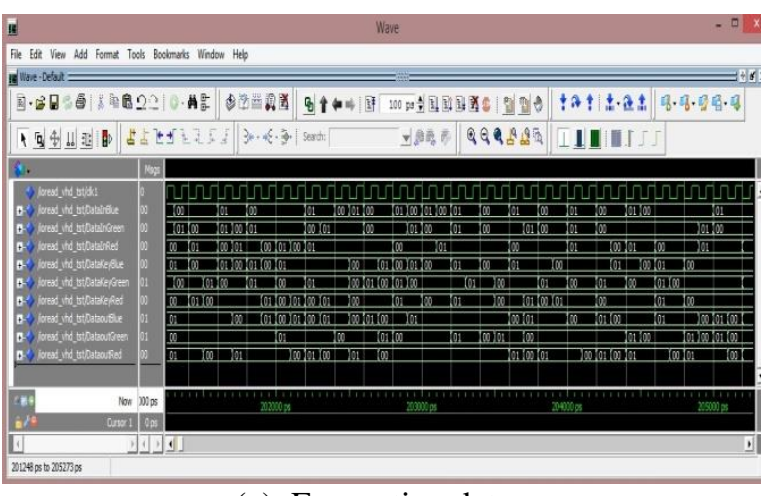

(a). Encryption data

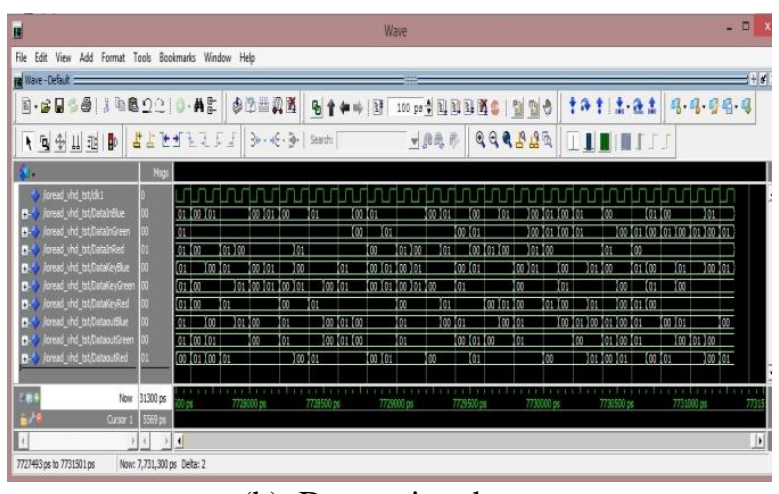

(b). Decryption data

Figure 8. Encryption and decryption data of image size 256x256

\section{CONCLUSION}

A color image encryption using a new chaotic map is simulated in software via Matlab, Altera Quartus Prime 17.0 Lite Edition I and ModelSim software tools then implemented in hardware via Cyclone V GX Starter Kit FPGA platform. The result shows the feasibility and effectiveness of the cryptosystem. As a typical application, the image encryption/decryption is to demonstrate and verify the operation of the cryptosystem hardware. The proposed scheme provides large key space, which is sensitive to slight change. The used security measurements demonstrated that the joint weaknesses existed in other encryption algorithms can be defeated by the proposed algorithm, and the security measurements show that the proposed algorithm robust against all types of attacks such as statistical, differential and brute-force attacks. The encryption execution time of the proposed encryption scheme is relatively fast and flexible to different applications.

\section{REFERENCES}

[1] Branislav J., "Synchronization Techniques for Chaotic Communication Systems," New Zealand: Springer, 2011.

[2] Qianxue Wang, Simin Yu, Chengqing Li, Jinhu L"u, Xiaole Fang, Christophe Guyeux, and Jacques M. Bahi,, "Theoretical Design and FPGA-Based Implementation of Higher-Dimensional Digital Chaotic Systems," IEEE TRANSACTIONS ON CIRCUITS AND SYSTEMS-I: REGULAR PARER, vol. 63, no. 3, p. 12, 2016.

[3] Bikash Baruah, Monjul Saikia,, "An FPGA Implementation of Chaos based Image Encryption and its Performance Analysis," International Journal of Computer Science and Network (IJCSN), vol. 5, no. 5, pp. 712-720, 2016.

[4] Safwan AI Assad, Ta Thi Kim Hue, Chu Van Lam, Thang Manh Hoang,, "Implementation of secure SPN chaosbased cryptosystem on FPGA," in IEEE International Symposium on Signal Processing and Information Technology (ISSPIT), Ho Chi Minh City, Vietnam, 2012. 
[5] Barlian Henryranu Prasetio, Eko Setiawan, Adharul Muttaqin,, "Image Encryption using Simple Algorithm on FPGA," TELKOMNIKA (Telecommunication Computing Electronics and Control), vol. 13, no. 4, pp. 1153-1161, 2015.

[6] Syed Shahzad Hussain Shah, Gulistan Raja,, "FPGA Implementation of Chaotic based AES Image Encryption Algorithm," in IEEE International Conference on Signal and Image Processing Applications (ICSIPA), Kuala Lumpur, Malaysia, 2015.

[7] Bhavna Sinha, Shubhendu Kumar, Chittaranjan Pradhan,, "Comparative Analysis of Color Image Encryption using 3D Chaotic Maps," in International Conference on Communication and Signal Processing,IEEE, India, 2016.

[8] Haider M. Al-Mashhadi, Iman Q. Abduljaleel,, "Color Image Encryption using Chaotic Maps Triangular Scrambling, with DNA Sequences," in International Conference on Current Research in Computer Science and Information Technology (ICCIT), Slemani, Iraq, 2017.

[9] A. A. R. a. H. H. F. Wafaa S. Sayed Ahmed G. Radwan, "Finite Precision Logistic Map between Computational Efficiency and Accuracy with Encryption Applications," Complexity, Hindawi., vol. 2017, p. 21, 2017.

[10] Hui Liu, Cong Jin,, "A Color Image Encryption Scheme Based on Arnold Scrambling and Quantum Chaotic," International Journal of Network Security, vol. 19, no. 3, pp. 347-357, 2017.

[11] Hamsa A. Abdullah, Hikmat N. Abdullah,, "A New Chaotic Map for Secure Transmission," TELKOMNIKA (Telecommunication Computing Electronics and Control), vol. 16, no. 3, pp. 1135-1142, 2018.

[12] Hikmat N. Abdullah, Hamsa A. Abdullah, "Two-level Secure Colored Image Transmission Using Novel Chaotic Map," in Second Al-Sadiq International Conference on Multidisciplinary in IT and Communication Science and Applications (2nd-AIC-MITC'17), Baghdad, Iraq, 2017.

[13] Hongyao Deng, Qingxin Zhu, Xiuli Song, Jingsong Tao,, "Chaos-Based Image Encryption Algorithm Using Decomposition," TELKOMNIKA (Telecommunication Computing Electronics and Control), vol. 12, no. 1, pp. 575-583, 2014.

[14] Alia Karim Abdul Hassan, "Proposed Hyperchaotic System for Image Encryption," International Journal of Advanced Computer Science and Applications (IJACSA), vol. 7, no. 1, 2016.

[15] Shivaputra, HS Sheshadri, V Lokesha, "A Naïve Visual Cryptographic Algorithm for the Transfer of Compressed Medical Images," Bulletin of Electrical Engineering and Informatics, vol. 5, no. 3, pp. 347-365, 2016.

[16] Borislav Stoyanov, Krasimir Kordov, "Image Encryption Using Chebyshev Map and Rotation Equation," Entropy, vol. 17, pp. 2117-2139, 2015.

[17] An American National Standard, "754-1985 - IEEE Standard for Binary Floating-Point Arithmetic," Standards Committee of the IEEE Computer Society, 1985.

[18] Ruisong Ye, "An Image Encryption Scheme with Efficient Permutation and Diffusion Processes," in In: Zhou M., Tan H. (eds) Advances in Computer Science and Education Applications, Communications in Computer and Information Science, Berlin, Heidelberg, Springer, 2011, pp. 32-39.

\section{BIOGRAPHIES OF AUTHORS}

Hamsa A. Abdullah was born in Baghdad, Iraq in 1984. She obtained her B.Sc. in Information
Engineering in 2005, M.Sc in Information Engineering in 2008 at Al-Nahrain University, Iraq.
She worked as a lecturer in the College of Information Engineering at Al-Nahrain University,
Iraq since 2008. She is interested in subjects Multimedia, Multimedia security transmission over
wireless communication systems, Pattern Recognition and Network Security.

